\title{
Mit dem Kompass in die Zukunft: Horizonte erweitern mit Tradition und Weitblick
}

\section{Anna Piepiorka Christine Schiller Katharina Treyer Julia Weber}

S. Karger Verlag GmbH, Redaktion KaRGER KompasS, Freiburg i.Br., Deutschland

Es gibt gute und schlechte Entwicklungen: Die Qualität liegt im Ermessen des Betrachters. «Früher war alles besser» ist eine Formel, die den Stillstand herbeisehnt und die Vergangenheit in ein romantisches Ambiente taucht - verlockend, wenn die Formulierung konkreter Ideen für die Zukunft schwerfällt. Die Medizin kann sich eine Absage an den Fortschritt jedoch nicht leisten. Ärzte versuchen qua ihrer Berufung Diagnostik, Therapie und Nachsorge ihrer Patienten stetig zu verbessern, bilden sich in diesen Bereichen kontinuierlich fort und dürfen die neuesten Forschungsergebnisse nicht auBer Acht lassen.

Uns von der Karger Kompass-Redaktion sind diese Anforderungen bewusst. Daher haben wir mit der Zeitschriftenreihe KARger Kompass vor allem mit Blick auf den praktischen Mediziner ein Konzept entwickelt, das eine moderne und praxisorientierte Interpretation der Forschung mit traditioneller Wissenschaftlichkeit verbindet - ganz in der Tradition des Karger Verlags, der seit Ende des 19. Jahrhunderts Fachwissen in Publikationen aufbereitet. Das Herzstück von KaRGER Kompass ist der «Wissenstransfer»: Darin werden aktuelle klinische und Grundlagenstudien von einem versierten, ständigen Fachbeirat auf Herz und Nieren geprüft und deren Nutzen kompakt und verständlich für die praktische
Arbeit aufbereitet. Aus dem Transfer ergibt sich auch eine Interpretationsanleitung, die gerade jungen Ärzten hilft, die richtigen Fragen zu stellen, Fallstricke zu erkennen und souverän Kritik zu üben. Um gerade den Interessen der Weiterbildungsassistenten bzw. angehenden Fachärzte näher zu kommen, hat die Redaktion ihre junge Zielgruppe selbst ins Boot geholt: Ab 2017 koordinieren die engagierten Jungmediziner PD Dr. med. Stephanie Joachim (Ophthalmologie, Bochum), Dr. med. Matthias Raspe (PNeumologie, Charité Berlin) und Dr. med. Nina Rosa Neuendorff (Onkologie, Charité Berlin) die neue Rubrik «Campus», in der sie in ausgewählten (Gast-)Beiträgen, Anleitungen und Interviews den fach- sowie berufspolitischen Nerv treffen. Thematisiert werden u.a. die Vereinbarkeit von Karriere und Familie, Vergütung, Weiterbildungsordnung, wissenschaftliches Schreiben etc; auch fachliches «Insiderwissen» findet seinen Platz, das oft nur mündlich «zwischen Tür und Angel» vermittelt wird. Damit rundet die Rubrik das Karger Kompass-Konzept ab, das mit dem «Wissenstransfer» über den «Campus» bis hin zum «Steckbrief Forschung» die Entwicklungen der medizinischen Zunft von morgen abbildet, indem er ihre Stimme von heute berücksichtigt. Weitere Rubriken ergänzen das Themenspektrum, das sich über die Dis- ziplinen Dermatologie, Onkologie, Ophthalmologie und Pneumologie erstreckt.

Dies ist unser konstruktiver Versuch, das zu formulieren und abzudecken, was wir als Bedürfnisse und Bedarf in der medizinischen Weiterbildung wahrnehmen: Reduktion komplexer Inhalte, mehr Verständlichkeit durch Informationen in deutscher Sprache, Plattform für die Belange junger Mediziner, fachspezifische und berufspolitische Themen etc. Dafür haben wir nicht nur qualitativ, sondern auch quantitativ aufgerüstet: Ab 2017 erscheint Karger Kompass DeRMATOlogie mit verstärktem Beirat (z.B. im Bereich Seniorendermatologie und Photodermatologie) und mit 4 statt 2 Ausgaben pro Jahr. Zudem bieten wir unseren Lesern erweiterte Inhalte und sinnvolle Kontextualisierungen auch über das Zeitschriftenformat hinaus auf unserer neuen FacebookSeite (www.facebook.com/kargerkompassdermatologie).

KaRGer Kompass, liebe Leser, ist unser Versuch, diesen Bedürfnissen entgegenzukommen. Wir als Redaktion stehen auf einer Seite der medizinischen Welt, deren Sinnhaftigkeit von den Interessen ihrer Leser abhängt. Sollten wir diesen Interessen in Ihren Augen noch nicht nah genug sein, bitten wir Sie um Ihre Meinung. Denn unsere Kompassnadel richtet sich nach Ihrem Sinne!

Ihre

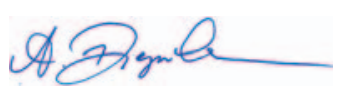

\section{Anna Piepiorka}

Projektmanagement

Karger Kompass -

Redaktion KaRGER Kompass

PNeUMologie Lektorat

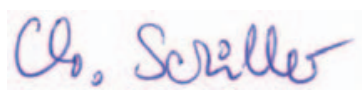

\section{Christine Schiller}

«Kaleidoskop»-Redaktion

Karger Kompass OnKologie

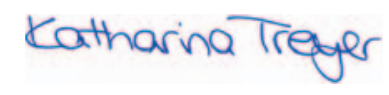

\section{Katharina Treyer}

Social Media / Facebook Redaktion Karger Kompass

Dermatologie \& Ophthalmologie

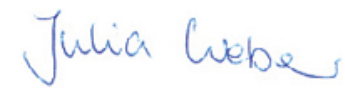

\section{Julia Weber}

«Campus» - Lektorat - Volontärin

\section{KARGER}

Fax +497614520714

information@karger.com

www.karger.com (c) 2017 S. Karger GmbH, Freiburg

Accessible online at:

www.karger.com/kkd
Katharina Treyer

S. Karger Verlag GmbH

Wilhelmstraße 20A, 79098 Freiburg, Deutschlan

k.treyer@karger.com 